\title{
Finding Common Interests
}

\author{
Hee Jeong Yoo \\ Department of Psychiatry, Seoul National University College of Medicine, Seoul National University Bundang Hospital, Seongnam, Korea
}

The Korean Academy of Child and Adolescent Psychiatry (KACAP) publishes this journal, which is mainly composed of child and adolescent psychiatrists with a variety of interests and professions. One of the journal's priorities is how to create a bridge between members who work in different disciplines of child and adolescent psychiatry, such as academic researchers and community-based clinical practitioners. Finding a common interest is the first step in building a good relationship while keeping each other's identity, as the journal should belong to all the members of the academy and be a place for communication. Finding and fostering common interests is also crucial to maintain continuing synergy between research findings and clinical practice applications.

To fulfill these objectives, our editorial office is planning several activities in 2022. First, we will conduct member surveys regarding the journal to make references for important decisions we will be making. After the Vision 2030 survey on the academy's 40th anniversary, it has been nine years since the academy formally heard the members' opinions. The survey will ask a variety of questions on the journal's identity, present, and future. The survey will seek feedback on more practical issues such as the journal's preferences for manuscript submission, importance of maintaining the paperform, and user-friendliness of manuscript processing. As a representative journal of child and adolescent psychiatry, we shall collect the most popular themes.

Second, the journal takes an active role in the discussion of clinically relevant and urgent issues. The Infant Comprehensive Evaluation for Neurodevelopmental Delay (ICEND), for example, is a comprehensive screening instrument for developmental delay and associated disorders from infancy to early childhood, and its validation research is reported in this issue. Early screening and differential diagnosis of develop- mental delay are therapeutically relevant issues not only in child psychiatry, but also in public health, pediatrics, and early education systems. Validation studies of diagnostic/screening devices, both new and existing, are welcome in our journal because they can be put to immediate use. In the same context, for the coming year, our journal is preparing special issues on practice guidelines for autism spectrum disorder and post-COVID-19 school mental health. I hope that our journal will pave the way for more direct connections between research and clinical practice.

Third, beginning last year, the editorial boards attempted to invite more international authors and reviewers by informing them of the journal's scope and vision. In this issue, authors from India have submitted a review article on the efficacy of a visual activity schedule intervention on attention deficit hyperactivity disorder. I am grateful to the international authors and reviewers, as such exchanges of interest from a global perspective would allow us to broaden and deepen our academic competence. If there are culturally sensitive issues or events in the field of child and adolescent mental health in each country, I hope you will consider submitting research to our journal.

It is hard to believe it has been two years since the pandemic began, with huge uncertainty and fear. However, I believe that, in any case, we should do the best we can. What I can do as a researcher and editor is establish and elaborate more diverse common interests in our field, fostering more consolidated interchange among researchers and clinicians via the platform of the Journal of KACAP. Besides the manuscripts themselves, any suggestions regarding new research areas, discussions of socially relevant topics, or research subjects for trainees are welcomed and fully considered. I hope you find our journal to be a reliable medium for mutual communication.

This is an Open Access article distributed under the terms of the Creative Common Attribution Non-Commercial License (https://creativecommons.org/licenses/by-nc/4.0) which permits unrestricted non-commercial use, distribution, and reproduction in any medium, provided the original work is properly cited. 